PROCEEDINGS OF THE

AMERICAN MATHEMATICAL SOCIETY

Volume 128, Number 1, Pages 307-313

$\mathrm{S}$ 0002-9939(99)05252-1

Article electronically published on May 6, 1999

\title{
INVOLUTIONS WITH $W(F)=1$
}

\author{
ZHI LÜ
}

(Communicated by Ralph Cohen)

\begin{abstract}
Let $\left(T, M^{n}\right)$ be a smooth involution on a closed $n$-dimensional manifold such that all Stiefel-Whitney classes of the tangent bundle to each component of the fixed point set $F$ of $\left(T, M^{n}\right)$ vanish in positive dimension. In this paper, we estimate the least possible lower bound of $\operatorname{dim} F$ if $\left(T, M^{n}\right)$ does not bound.
\end{abstract}

\section{INTRODUCTION}

Let $\left(T, M^{n}\right)$ be a smooth involution on a closed $n$-dimensional manifold. It is very well known that the fixed point set $F$ of $\left(T, M^{n}\right)$ is the finite disjoint union of closed submanifolds of $M^{n}$. Let $F^{n-k}$ denote the union of those components of the fixed point set $F$ having dimension $n-k$ and let $\nu^{k}$ denote the normal bundle of $F^{n-k}$ in $M^{n}$. By $\operatorname{dim} F$ we mean the dimension of the highest dimensional non-empty component of the fixed point set $F$.

In [1] Conner and Floyd examined involutions $\left(T, M^{n}\right)$ having the property $W\left(F^{n-k}\right)=1$ for all $k$ (here $W$ denotes the total Stiefel-Whitney class), and proved that if all the components of $F$ have same dimension, then $\left(T, M^{n}\right)$ bounds equivariantly. At the same time, they also gave an example and showed that the result fails if the dimensions of the components of $F$ are allowed to vary. Later on, Kosniowski and Stong in [4] discussed the problem again, and obtained that if $\left(T, M^{n}\right)$ is an involution with $W\left(F^{n-k}\right)=1$ for all $k$, then the equivariant bordism class of $\left(T, M^{n}\right)$ is determined by the bordism class of $M^{n}$. In this paper, we prove that if $n>2 \operatorname{dim} F$, then $\left(T, M^{n}\right)$ with $W\left(F^{n-k}\right)=1$ for all $k$ bounds equivariantly, and see from an example that $\frac{n}{2}$ is exactly the least possible lower bound of $\operatorname{dim} F$ if $\left(T, M^{n}\right)$ with $W\left(F^{n-k}\right)=1$ for all $k$ does not bound. Our main result is stated as follows.

Theorem A. Suppose $\left(T, M^{n}\right)$ is a smooth involution such that $W\left(F^{n-k}\right)=1$ for all $k$. If $n>2 \operatorname{dim} F$, then $\left(T, M^{n}\right)$ bounds equivariantly.

Remark. It should be pointed out that if we change the condition $n>2 \operatorname{dim} F$ into $n \leq 2 \operatorname{dim} F$ in Theorem A, then the result fails. For example, an involution

Received by the editors March 24, 1998.

1991 Mathematics Subject Classification. Primary 57R85; Secondary 57R90.

Key words and phrases. Involution, fixed point set, symmetric polynomial function, bordism.

This work is supported by Youthful Foundation of Tsinghua University and the Japanese Government Scholarship.

(C)1999 American Mathematical Society 
$(T, R P(2))$ defined by

$$
T:\left[x_{0}, x_{1}, x_{2}\right] \longrightarrow\left[-x_{0}, x_{1}, x_{2}\right]
$$

fixing $F=* \sqcup R P(1)$ (* denotes a point) does not bound, but $2 \operatorname{dim} F=\operatorname{dim} R P(2)$.

This means that if $\left(T, M^{n}\right)$ with $W\left(F^{n-k}\right)=1$ for all $k$ does not bound, then the least possible lower bound of $\operatorname{dim} F$ is exactly $n / 2$, and thus the result of Theorem $\mathrm{A}$ is best possible.

In fact, the condition that $W\left(F^{n-k}\right)=1$ for all $k$ in Theorem $\mathrm{A}$ can also be changed into more general case. We state the result as follows.

Theorem B. Suppose that $\left(T, M^{n}\right)$ is a smooth involution such that $W\left(F^{n-k}\right)=1$ for all $k \leq \frac{3}{5} n$, but not necessarily for $k>\frac{3}{5} n$. If $n>2 \operatorname{dim} F$, then $\left(T, M^{n}\right)$ bounds equivariantly.

Throughout this paper, the coefficient group is $\mathbb{Z}_{2}$. Let $[N]$ denote the fundamental homology class of closed smooth manifold $N$, and $\{N\}$ the unoriented bordism class of $N . \sigma_{i}\left(x_{1}, \cdots, x_{n}\right)$ denotes the $i$-th elementary symmetric function $\sum x_{1} \cdots x_{i}$, and $S_{\omega}\left(x_{1}, \cdots, x_{n}\right)=\sum x_{1}^{i_{1}} \cdots x_{r}^{i_{r}}$ denotes the usual smallest symmetric polynomial containing the given monomial, where $\omega=\left(i_{1}, \cdots, i_{r}\right)$ is a partition of $|\omega|=i_{1}+\cdots+i_{r}$.

\section{Preliminaries}

First, we introduce an important formula given by Kosniowski and Stong [4] for the calculation of the Stiefel-Whitney numbers of $M^{n}$ in terms of the fixed point data $\nu \rightarrow F=\bigsqcup_{k} \nu^{k} \rightarrow F^{n-k}$. That is the following

Proposition 1 (Kosniowski and Stong). If $f\left(x_{1}, \cdots, x_{n}\right)$ is any symmetric polynomial over $\mathbb{Z}_{2}$ in $n$ variables of degree at most $n$, then

$$
f\left(x_{1}, \cdots, x_{n}\right)\left[M^{n}\right]=\sum_{k} \frac{f\left(1+y_{1}, \cdots, 1+y_{k}, z_{1}, \cdots, z_{n-k}\right)}{\prod_{i=1}^{k}\left(1+y_{i}\right)}\left[F^{n-k}\right]
$$

where the expressions are evaluated by replacing the elementary symmetric functions $\sigma_{i}(x), \sigma_{i}(y)$, and $\sigma_{i}(z)$ by the Stiefel-Whitney classes $W_{i}\left(M^{n}\right), W_{i}\left(\nu^{k}\right)$, and $W_{i}\left(F^{n-k}\right)$ respectively, and taking the value of the resulting cohomology class on the fundamental homology class of $M^{n}$ or $F^{n-k}$.

Notice that in this paper our interest mainly focuses on discussing the involution $\left(M^{n}, T\right)$ having the property $W\left(F^{n-k}\right)=1$ for all $k$. It is easy to see that if $W\left(F^{n-k}\right)=1$ for all $k$, then we may choose $z_{i}=0$ in Proposition 1 from the splitting principle. Thus we have ( here we also give a simple algebric proof)

Corollary 1. Suppose $\left(T, M^{n}\right)$ is an involution with $W\left(F^{n-k}\right)=1$ for all $k$. Then

$$
f\left(x_{1}, \cdots, x_{n}\right)\left[M^{n}\right]=\sum_{k} \frac{f(1+y_{1}, \cdots, 1+y_{k}, \overbrace{0, \cdots, 0}^{n-k})}{\left(1+y_{1}\right) \cdots\left(1+y_{k}\right)}\left[F^{n-k}\right]
$$

for all the symmetric polynomials $f\left(x_{1}, \cdots, x_{n}\right)$ in $n$ variable of degree at most $n$. 
Proof. From the fact [4, p.317, Lemma], that for each $k$,

$$
\begin{aligned}
\sigma_{i}\left(1+y_{1}, \cdots, 1+y_{k}, z_{1}, \cdots, z_{n-k}\right) \\
\quad=\sum_{p+q \leq i}\left(\begin{array}{c}
k-p \\
i-p-q
\end{array}\right) \sigma_{p}\left(y_{1}, \cdots, y_{k}\right) \sigma_{q}\left(z_{1}, \cdots, z_{n-k}\right),
\end{aligned}
$$

it is easy to see that if $W\left(F^{n-k}\right)=1$, i.e., $\sigma_{q}\left(z_{1}, \cdots, z_{n-k}\right)=0$ for all $q \geq 1$, then

$$
\begin{aligned}
& \sigma_{i}\left(1+y_{1}, \cdots, 1+y_{k}, z_{1}, \cdots, z_{n-k}\right) \\
= & \sum_{p \leq i}\left(\begin{array}{c}
k-p \\
i-p
\end{array}\right) \sigma_{p}\left(y_{1}, \cdots, y_{k}\right) \\
= & \sigma_{i}(1+y_{1}, \cdots, 1+y_{k}, \underbrace{0, \cdots, 0}_{n-k}) .
\end{aligned}
$$

On the other hand, it is very well known that for the symmetric polynomial $f\left(x_{1}, \cdots, x_{n}\right)$, there must exist a polynomial $\phi\left(t_{1}, \cdots, t_{n}\right)$ in $n$ variables such that

$$
\begin{aligned}
& f\left(1+y_{1}, \cdots, 1+y_{k}, z_{1}, \cdots, z_{n-k}\right) \\
& =\phi\left(\sigma_{1}\left(1+y_{1}, \cdots, 1+y_{k}, z_{1}, \cdots, z_{n-k}\right), \cdots,\right. \\
& \left.\sigma_{n}\left(1+y_{1}, \cdots, 1+y_{k}, z_{1}, \cdots, z_{n-k}\right)\right) .
\end{aligned}
$$

Combining (2.1) and (2.2), it at once follows that if $\sigma_{q}\left(z_{1}, \cdots, z_{n-k}\right)=0$ for all $q \geq 1$, then

$$
f\left(1+y_{1}, \cdots, 1+y_{k}, z_{1}, \cdots, z_{n-k}\right)=f(1+y_{1}, \cdots, 1+y_{k}, \underbrace{0, \cdots, 0}_{n-k}),
$$

and the corollary thus holds by Proposition 1. This completes the proof.

Next, in order to prove our main theorem, we also need the following result due to Kosniowski and Stong (see [4, p. 326, Proposition]), and its proof is included here for local completeness.

Proposition 2. Suppose that $\left(T, M^{n}\right)$ is a smooth involution having the property $W\left(F^{n-k}\right)=1$ for all $k$. Then $\left(T, M^{n}\right)$ bounds equivariantly if and only if $M^{n}$ bounds.

Proof. First, it follows immediately that $M^{n}$ bounds if $\left(T, M^{n}\right)$ bounds equivariantly.

Conversely, for each $k$, since $W\left(F^{n-k}\right)=1$, the unoriented bordism class of the normal bundle $\nu^{k} \rightarrow F^{n-k}$ is determined by the numbers

$$
S_{\omega}\left(\nu^{k}\right)\left[F^{n-k}\right]
$$

where $\omega=\left(i_{1}, \cdots, i_{r}\right)$ is any a partition of $n-k$. In particular, if $r>k$, then $S_{\omega}\left(\nu^{k}\right)\left[F^{n-k}\right]=0$ for $\nu^{k} \rightarrow F^{n-k}$ is a $k$-plane bundle. Let $\omega=\left(i_{1}, \cdots, i_{r}\right)$ with $r \leq k$ be any partition of $n-k$, and take

$$
f_{\omega}\left(x_{1}, \cdots, x_{n}\right)=\sum\left(1+x_{1}\right)^{i_{1}} x_{1} \cdots\left(1+x_{r}\right)^{i_{r}} x_{r} x_{r+1} \cdots x_{k} .
$$

Then

$$
\frac{f_{\omega}(1+y_{1}, \cdots, 1+y_{k}, \overbrace{0, \cdots, 0}^{n-k})}{\left(1+y_{1}\right) \cdots\left(1+y_{k}\right)}=\sum y_{1}^{i_{1}} \cdots y_{r}^{i_{r}}=S_{\omega}\left(y_{1}, \cdots, y_{k}\right),
$$


but for $h<k$,

$$
f_{\omega}(1+y_{1}, \cdots, 1+y_{h}, \underbrace{0, \cdots, 0}_{n-h})=0
$$

and for $h>k$,

$$
\min \left\{\operatorname{deg} f_{\omega}(1+y_{1}, \cdots, 1+y_{h}, \underbrace{0, \cdots, 0}_{n-h})\right\} \geq n-k>n-h
$$

(here $\min \left\{\operatorname{deg} f_{\omega}(1+y_{1}, \cdots, 1+y_{h}, \underbrace{0, \cdots, 0}_{n-h})\right\}$ denotes the degree of the least degree term in $f_{\omega}(1+y_{1}, \cdots, 1+y_{h}, \underbrace{0, \cdots, 0)}_{n-h})$; hence by Corollary 1 , we have

$$
S_{\omega}\left(\nu^{k}\right)\left[F^{n-k}\right]=f_{\omega}(x)\left[M^{n}\right]=S_{(i_{1}+1, \cdots, i_{r}+1, \underbrace{1, \cdots, 1}_{k-r}}^{1, \cdots}\left\{M^{n}\right\} .
$$

Note that, for each component $F^{n-h}$ with $h>k$,

$$
\frac{f_{\omega}(1+y_{1}, \cdots, 1+y_{h}, \overbrace{0, \cdots, 0}^{n-h})}{\left(1+y_{1}\right) \cdots\left(1+y_{h}\right)}\left[F^{n-h}\right]=0
$$

by (2.3). From (2.4), it follows at once that if $M^{n}$ bounds, then $\nu^{k} \rightarrow F^{n-k}$ bounds for each $k$. Again by [3, Theorem (25.2)], we obtain that $\left(T, M^{n}\right)$ bounds equivariantly. This completes the proof of the proposition.

From the proof of Proposition 2, we easily see

Corollary 2. Let $\left(T, M^{n}\right)$ be an involution with $W\left(F^{n-k}\right)=1$ for all $k$. Then $M^{n}$ is decomposable if and only if the normal bundle of the $(n-1)$-dimensional fixed point set component bounds.

\section{Proofs of Theorem A And Theorem B}

As in defined in [5], we say that $\omega=\left(i_{1}, \cdots, i_{s}\right)$ is a non-dyadic partition of the positive integer $l$ if $|\omega|=i_{1}+\cdots+i_{s}=l$ and the none of $i_{\alpha} \in \omega$ is of the form $2^{p}-1$.

Now we begin with the proof of Theorem A.

Proof of Theorem $A$. Let $\omega=\left(2 i_{1}+1, \cdots, 2 i_{s}+1,2 j_{1}, \cdots, 2 j_{t}\right)$ be any a non-dyadic partition of $n$. Take

$$
\begin{aligned}
& f_{\omega}\left(x_{1}, \cdots, x_{n}\right) \\
= & \sum\left(1+x_{1}\right)^{i_{1}+1} x_{1}^{i_{1}} \cdots\left(1+x_{s}\right)^{i_{s}+1} x_{s}^{i_{s}}\left(1+x_{s+1}\right)^{j_{1}} x_{s+1}^{j_{1}} \cdots\left(1+x_{s+t}\right)^{j_{t}} x_{s+t}^{j_{t}},
\end{aligned}
$$

since $W\left(F^{n-k}\right)=1$ for all $k$; then

$$
S_{\omega}\left\{M^{n}\right\}=\sum_{k} \frac{f_{\omega}(1+y_{1}, \cdots, 1+y_{k}, \overbrace{0, \cdots, 0}^{n-k})}{\left(1+y_{1}\right) \cdots\left(1+y_{k}\right)}\left[F^{n-k}\right]
$$

by Corollary 1 . When $k<s+t$, it is obvious that

$$
f_{\omega}(1+y_{1}, \cdots, 1+y_{k}, \underbrace{0, \cdots, 0}_{n-k})=0,
$$


and thus

$$
\sum_{k<s+t} \frac{f_{\omega}(1+y_{1}, \cdots, 1+y_{k}, \overbrace{0, \cdots, 0}^{n-k})}{\left(1+y_{1}\right) \cdots\left(1+y_{k}\right)}\left[F^{n-k}\right]=0 .
$$

When $k \geq s+t$, we have

$$
\begin{aligned}
& f_{\omega}(1+y_{1}, \cdots, 1+y_{k}, \underbrace{0, \cdots, 0}_{n-k}) \\
= & \sum y_{1}^{i_{1}+1}\left(1+y_{1}\right)^{i_{1}} \cdots y_{s}^{i_{s}+1}\left(1+y_{s}\right)^{i_{s}} y_{s+1}^{j_{1}}\left(1+y_{s+1}\right)^{j_{1}} \cdots y_{s+t}^{j_{t}}\left(1+y_{s+t}\right)^{j_{t}},
\end{aligned}
$$

and thus

$$
\begin{aligned}
\min & \left\{\operatorname{deg} f_{\omega}(1+y_{1}, \cdots, 1+y_{k}, \underbrace{0, \cdots, 0}_{n-k})\right\} \\
& \geq\left(i_{1}+1\right)+\cdots+\left(i_{s}+1\right)+j_{1}+\cdots+j_{t} \\
& =\frac{n+s}{2} \\
& \geq \frac{n}{2} .
\end{aligned}
$$

Since $n>2 \operatorname{dim} F$, i.e., $2 k>n$ for all $k$, we have

$$
\sum_{k \geq s+t} \frac{f_{\omega}(1+y_{1}, \cdots, 1+y_{k}, \overbrace{0, \cdots, 0}^{n-k})}{\left(1+y_{1}\right) \cdots\left(1+y_{k}\right)}\left[F^{n-k}\right]=0 .
$$

Hence it follows from (3.1), (3.2) and (3.3) that $S_{\omega}\left\{M^{n}\right\}=0$, i.e., $M^{n}$ bounds. Again by Proposition 2, we obtain that $\left(T, M^{n}\right)$ bounds equivariantly. This completes the proof of Theorem A.

The proof of Theorem B is mainly based on the following lemma and the Boardman 5/2-Theorem (see [6]). It should be pointed out that Kosniowski and Stong [4] obtained the stronger Boardman 5/2-Theorem, which here we will use.

Lemma 3.1. Suppose $\left(T, M^{n}\right)$ is a smooth involution such that $W\left(F^{n-k}\right)=1$ for all $k \leq \frac{3}{5} n$, but not necessarily for $k>\frac{3}{5} n$. If $n>2 \operatorname{dim} F$, then $M^{n}$ bounds.

Proof. Let $\omega=\left(2 i_{1}+1, \cdots, 2 i_{s}+1,2 j_{1}, \cdots, 2 j_{t}\right)$ be any a non-dyadic partition of $n$. Choose

$$
\begin{aligned}
& f_{\omega}\left(x_{1}, \cdots, x_{n}\right) \\
= & \sum\left(1+x_{1}\right)^{i_{1}+1} x_{1}^{i_{1}} \cdots\left(1+x_{s}\right)^{i_{s}+1} x_{s}^{i_{s}}\left(1+x_{s+1}\right)^{j_{1}} x_{s+1}^{j_{1}} \cdots\left(1+x_{s+t}\right)^{j_{t}} x_{s+t}^{j_{t}} ;
\end{aligned}
$$

by Proposition 1 and the proof of Corollary 1, we have

$$
\begin{aligned}
& S_{\omega}\left\{M^{n}\right\}=f_{\omega}\left(x_{1}, \cdots, x_{n}\right)\left[M^{n}\right] \\
= & \sum_{k \leq \frac{3}{5} n} \frac{f_{\omega}(1+y_{1}, \cdots, 1+y_{k}, \overbrace{0, \cdots, 0}^{n-k})}{\left(1+y_{1}\right) \cdots\left(1+y_{k}\right)}\left[F^{n-k}\right] \\
& +\sum_{k>\frac{3}{5} n} \frac{f_{\omega}\left(1+y_{1}, \cdots, 1+y_{k}, z_{1}, \cdots, z_{n-k}\right)}{\left(1+y_{1}\right) \cdots\left(1+y_{k}\right)}\left[F^{n-k}\right] .
\end{aligned}
$$


As in the proof of Theorem A, first we have

$$
\sum_{k \leq \frac{3}{5} n} \frac{f_{\omega}(1+y_{1}, \cdots, 1+y_{k}, \overbrace{0, \cdots, 0}^{n-k})}{\left(1+y_{1}\right) \cdots\left(1+y_{k}\right)}\left[F^{n-k}\right]=0 .
$$

Notice that $k>n / 2$ for all $k$ since $n>2 \operatorname{dim} F$. Next, since $\omega$ is a non-dyadic partition of $n$, we have $i_{\alpha} \geq 2(\alpha=1, \cdots, s)$, and thus

$$
n=\sum_{\alpha=1}^{s}\left(2 i_{\alpha}+1\right)+\sum_{\beta=1}^{t} 2 j_{\beta} \geq \sum_{\alpha=1}^{s}\left(2 i_{\alpha}+1\right) \geq 5 s,
$$

i.e., $s \leq \frac{1}{5} n$. Furthermore, for $k>\frac{3}{5} n$,

$$
\begin{aligned}
\min \left\{\operatorname{deg} f_{\omega}\left(1+y_{1}, \cdots, 1+y_{k}, z_{1}, \cdots, z_{n-k}\right)\right\} & \geq i_{1}+\cdots+i_{s}+j_{1}+\cdots+j_{t} \\
& =\frac{n-s}{2} \\
& \geq \frac{2}{5} n \\
& >n-k .
\end{aligned}
$$

Hence

$$
\sum_{k>\frac{3}{5} n} \frac{f_{\omega}\left(1+y_{1}, \cdots, 1+y_{k}, z_{1}, \cdots, z_{n-k}\right)}{\left(1+y_{1}\right) \cdots\left(1+y_{k}\right)}\left[F^{n-k}\right]=0 .
$$

From (3.4), (3.5) and (3.6), it follows at once that $S_{\omega}\left\{M^{n}\right\}=0$, i.e., $M^{n}$ bounds. This completes the proof of the lemma.

Being given an involution $\left(T, M^{n}\right)$, let $\Gamma^{1}\left(M^{n}\right)$ denote an $(n+1)$-dimensional manifold formed from the product $S^{1} \times M^{n}$ of the circle with $M^{n}$ by identifying $(z, x)$ with $(-z, T x)$, with the involution $T_{1}$ on $\Gamma^{1}\left(M^{n}\right)$ induced by $(z, x) \rightarrow(\bar{z}, x)$, as defined in [3]. Let $\left(T_{0}, \Gamma^{0}\left(M^{n}\right)\right)=\left(T, M^{n}\right)$, and let $\left(T_{r}, \Gamma^{r}\left(M^{n}\right)\right)$ be the $r$-th iterate of $\left(T, M^{n}\right)$. Thus a sequence of involutions $\left(T_{r}, \Gamma^{r}\left(M^{n}\right)\right)$ is constructed. In particular, we also know from [3] that the normal bundle to the fixed point set of $\left(T_{r}, \Gamma^{r}\left(M^{n}\right)\right)$ is

$$
\bigsqcup_{k}\left(\nu^{k} \oplus r \mathbb{R} \rightarrow F^{n-k}\right) \sqcup\left(\bigsqcup_{j=0}^{r-1}(r-j) \mathbb{R} \rightarrow \Gamma^{j}\left(M^{n}\right)\right)
$$

where $\nu \rightarrow F=\bigsqcup_{k}\left(\nu^{k} \rightarrow F^{n-k}\right)$ is the original normal bundle to $F$ in $M^{n}$.

Let $M O_{m}(B O(p))$ denote the unoriented bordism group of real $p$-dimensional plane bundles over closed smooth $m$-dimensional manifolds.

Proof of Theorem B. Consider the involution $\left(T_{1}, \Gamma^{1}\left(M^{n}\right)\right)$ with the fixed point data $\bigsqcup_{k}\left(\nu^{k} \oplus \mathbb{R} \rightarrow F^{n-k}\right) \sqcup\left(\mathbb{R} \rightarrow M^{n}\right)$. Since $M^{n}$ bounds (by Lemma 3.1), we have that the fixed data of $\left(T_{1}, \Gamma^{1}\left(M^{n}\right)\right)$ is bordant to $\bigsqcup_{k}\left(\nu^{k} \oplus \mathbb{R} \rightarrow F^{n-k}\right)$. Again by Lemma 3.1, we obtain that $\Gamma^{1}\left(M^{n}\right)$ bounds. Continuing this process, it is easy to see that, for each $r \geq 1$, the fixed data of $\left(T_{r}, \Gamma^{r}\left(M^{n}\right)\right)$ is bordant to $\bigsqcup_{k}\left(\nu^{k} \oplus r \mathbb{R} \rightarrow F^{n-k}\right)$, and $\Gamma^{r}\left(M^{n}\right)$ bounds by Lemma 3.1. The stronger Boardman $5 / 2$-Theorem makes $\left(T_{r}, \Gamma^{r}\left(M^{n}\right)\right)$ bound for large $r$ (e.g., choose $r \geq\left[\frac{1}{2} \operatorname{dim} F\right]+$ 
1) and thus for each $k$, the bordism class of $\nu^{k} \oplus r \mathbb{R} \rightarrow F^{n-k}$ is equal to 0 in $M O_{n-k}(B O(k+r))$. Since

$$
\oplus r \mathbb{R}: M O_{n-k}(B O(k)) \rightarrow M O_{n-k}(B O(k+r))
$$

is a monomorphism, this implies that for each $k, \nu^{k} \rightarrow F^{n-k}$ bounds, i.e., $\nu \rightarrow F=$ $\bigsqcup_{k}\left(\nu^{k} \rightarrow F^{n-k}\right)$ bounds. Thus $\left(T, M^{n}\right)$ bounds equivariantly. This completes the proof of Theorem B.

\section{ACKNOWLEDGMENT}

The author wishes to thank the referee for his valuable suggestions.

\section{REFERENCES}

[1] P.E.Conner and E.E.Floyd, Differentiable periodic maps, Springer, Berlin, 1964. MR 31:750

[2] P.E.Conner and E.E.Floyd, Fibring within a cobordism class, Mich. Math. J., 12(1965), 3347. MR 31:4038

[3] P.E.Conner, Differentiable periodic maps, 2nd ed., Lecture Notes in Math., 738 (Springer,Berlin,1979). MR 81f:57018

[4] C.Kosniowski and R.E.Stong, Involutions and characteristic numbers, Topology, 17(1978), 309-330. MR 82a:57036

[5] R.E.Stong, Notes on cobordism theory, Princeton University Press, Princeton, N. J.,1968. MR 40:2108

[6] J.M.Boardman, On manifolds with involution, Bull. Amer. Math. Soc., 73(1968),136-138. MR 34:5093

Department of Applied Mathematics, Tsinghua University, Beijing, 100084, People's RePublic of China

Current address: Graduate School of Mathematical Sciences, University of Tokyo, 3-8-1, Komaba, Meguro-ku, Tokyo 153-8914, Japan

E-mail address: zlu@ms326kaz.ms.u-tokyo.ac.jp 